\title{
OsABCB14 functions in auxin transport and iron homeostasis in rice (Oryza
} sativa.L)

YanXia Xu ${ }^{1, \dagger}$, SaiNa Zhang ${ }^{1, \dagger}$, HaiPeng Guo ${ }^{1}$, SuiKang Wang ${ }^{1}$, LiGen $\mathrm{Xu}^{1}$, ChuanYou $\mathrm{Li}^{3}$, Qian Qian ${ }^{4}$, Fan Chen ${ }^{5}$, Markus Geisler ${ }^{2}$, YanHua Qi ${ }^{* 1}$ and De An Jiang ${ }^{* 1}$

${ }^{1}$ State Key Laboratory of Plant Physiology and Biochemistry, College of Life Sciences, Zhejiang University, Hangzhou, China;

2 Department of Biology - Plant Biology, University of Fribourg, Rue Albert-Gockel 3, CH-1700 Fribourg, Switzerland

3 State Key Laboratory of Plant Genomics, National Center for Plant Gene Research, Institute of Genetics and Developmental Biology, Chinese Academy of Sciences, Beijing, China.

${ }^{4}$ State Key Laboratory of Rice Biology, China National Rice Research Institute, Chinese Academy of Agricultural Sciences, Hangzhou, China, and

${ }^{5}$ State Key Laboratory of Molecular Developmental Biology, Institute of Genetics and Developmental Biology, Chinese Academy of Sciences, Beijing, China.

* For correspondence:

YanHua Qi,

Tel: +8657188981355 
E-mail:qyhjp@zju.edu.cn

De An Jiang,

Tel: +8657188206461

Email: dajiang@zju.edu.cn

$\dagger$ These authors contributed equally to this work.

\section{RUNNING TITLE: Auxin transport by rice ABCB14}

\section{SUMMARY}

Members of the ATP Binding Cassette B/Multidrug-resistance/P-glycoprotein (ABCB/MDR/PGP) subfamily were shown to function primarily in auxin transport. However, none of rice $\mathrm{ABCB}$ transporters have been functionally characterized. Here, we describe that a knock-down of $O s A B C B 14$ confers decreased auxin concentrations and polar auxin transport rates, conferring insensitivity to 2,4-D and IAA. OsABCB14 displays enhanced specific auxin influx activity in yeast and protoplasts prepared from rice knock-down alleles. OsABCB14 is localized at the plasma membrane pointing to an important directionality under physiological conditions. osabcbl4 mutants were surprisingly found to be insensitive to iron deficiency treatment $(-\mathrm{Fe})$. Their Fe concentration is higher and up-regulation of Fe-deficiency responsive genes is lower in osabcb14 mutants than in wild type rice, Nipponbare (NIP). Taken together, our results strongly support the role of $O s A B C B 14$ as an 
auxin influx transporter involved in iron homeostasis. The functional characterization of OsABCB14 provides insights in monocot auxin transport and its relationship to Fe nutrition.

KEY WORDS: OsABCB14, polar auxin transport, iron homeostasis, rice (Oryza sativa.L)

\section{INTRODUCTION}

Auxin regulates many aspects of plant growth and development (Woodward and Bartel, 2005; Leyser, 2006; Teale et al., 2006). Auxin is thought to be synthesized in young apical tissues and then to enter basal tissues including the maturing stem and the roots by a polar transport system. The distribution of auxin and the formation of auxin gradients in the tissues are supposed to be directed by the activity of members of three families: the PIN-FORMED (PIN) family, AUXIN RESISTANT1/LIKE AUX1 (AUX1/LAX) family and ATP Binding Cassette B (ABCB) subfamily (Cho and Cho, 2012). Members of the long-looped PIN family seem to be responsible for the auxin efflux from cells (Krecek et al., 2009), whereas AUX/LAX family charge the auxin influx into cells (Peret et al., 2012), while the auxin transport directionality of $\mathrm{ABCB}$ subfamily seems to depend on cytoplasmic auxin concentration (Yang and Murphy, 2009; Abel and Theologis, 2010; Peer et al., 2011).

The ABCB subfamily is the second largest ATP-binding cassette $(\mathrm{ABC})$ protein subfamily and the largest full-molecule ABC transporter subfamily in plants (Rea 2007). ABCB's function in plants was first reported in the model plant Arabidopsis thaliana (Sidler et al., 
1998). For now, 5 ABCB genes in Arabidopsis (AtABCB1, AtABCB4, AtABCB14, AtABCB19 and $A t A B C B 21$ ) have been functionally characterized. AtABCB1 and AtABCB19 (a close homolog of AtABCB1) were demonstrated to function as auxin efflux transporters (Sidler et al., 1998; Noh et al., 2001; Geisler et al., 2003; Geisler et al., 2005; Cho et al., 2007; Wang et al., 2013). AtABCB4 and AtABCB21 (a close homolog of AtABCB4) were reported to have facultative auxin transport function, depending on auxin concentrations - auxin uptake activity at low auxin concentrations and invert to export activity at high auxin concentrations (Terasaka et al., 2005; Yang and Murphy, 2009; Kamimoto et al., 2012; Kubes et al., 2012). Additionally, AtABCB14 is a malate uptake transporter and plays an essential role under stress conditions (Lee et al., 2008). Studies of ABCBs in other species showed that ABCBs also able to transport secondary metabolites (Shitan et al., 2003) and to regulate aluminum tolerance and calcium homeostasis (Sasaki et al., 2002).

In Arabidopsis, evidences have been provided that the members of PIN and ABCB protein families regulate auxin transport coordinately and independently (Noh et al., 2003; Bandyopadhyay et al., 2007; Blakeslee et al., 2007; Mravec et al., 2008; Titapiwatanakun et al., 2009). Some studies also focused on the interaction between ABCBs and the immunophilin-like FKBP42, TWISTED DWARF1 (TWD1), and the protein phosphorylation of ABCBs. AtABCB1 was reported to be substrate of PINOID (PID), an AGC kinase. TWD1 interacts with $\mathrm{PID}$, and directs phosphorylation of $\mathrm{ABCB} 1$ in a regulatory linker domain that alters ABCB1-mediated auxin transport activity (Geisler et al., 2003; Geisler et al., 2004; Bouchard et al., 2006; Bailly et al., 2008; Henrichs et al., 2012). AtABCB19 also can be 
phosphorylated by PHOTOTROPIN 1 (phot1), a plasma membrane serine-threonine protein kinase involved in blue-light responses, to inhibit its efflux activity (Christie et al., 2011). All these above results were mainly established using dicotyledonous Arabidopsis as a model system, but the role of $\mathrm{ABCBs}$ is unclear in monocotyledons.

Several studies indicate a close interrelationship between auxin and iron ion homeostasis: auxin has been already reported since the 80 s to be implicated in iron deficiency-induced adaptive responses (Landsberg, 1981). Sunflower roots, were shown to own higher levels of auxin under Fe-deficient condition than Fe-sufficient (Römheld and Marschner, 1986). Reductions of auxin transport or auxin sensitivity all inhibited formation of root hair in response to Fe-deficiency (Schikora and Schmidt, 2001). Further, the influence of plant hormones on Fe uptake by strategy I plants was summarized in Romera et al. (2007). Based on reports from recent years, $\mathrm{Fe}$ deficiency can trigger an over-accumulation of indole-3-acetic acid (IAA) and Arabidopsis auxin transporter auxl-7 mutant plants exhibit reduced ferric chelate reductase activity under low Fe (Chen et al., 2010). Thus, the auxin transporter AtAUX1 is thought to mainly direct the root-ward auxin stream into lateral roots to integrate local Fe nutritional status (Giehl et al., 2012). In Malus xiaojinensis, it is also indicated that iron deficiency-induced physiological responses are mediated by systemic auxin signaling (Wu et al., 2012). We also reported that auxin response factor, OsARF12, regulates root elongation and affects in iron homeostasis in rice (Qi et al., 2012). However, the molecular mechanism of auxin in the regulation of iron deficiency responses still maintains unclear in monocot, needs to be systematically investigated further. 
It is important to note that, although rice is an important food crop, there are no reports on its ABCBs. To test whether $\mathrm{ABCB}$ also play an important role in rice, we investigated the behavior of $O S A B C B 14$ gene in rice. OsABCB14 is shown to function in cellular auxin uptake and iron homeostasis in rice.

\section{RESULTS:}

\section{Identification of osabcb14 mutants and complemented transgenic lines}

We obtained two TOS17 insertional lines in the $O s A B C B 14$ gene from the Rice Genome Resource Center in Japan. Using PCR analysis and sequencing, we confirmed that TOS17 had been inserted into the $8^{\text {th }}$ exon of the $O s A B C B 14$ gene at $o s a b c b 14-1$, and $7^{\text {th }}$ intron of OsABCB14 in osabcb14-2, respectively (Figure 1a). Both mutant lines are homozygous (Figure 1b). In addition, we overexpressed $O s A B C B 14$ in $o s a b c b 14-1$ and $o s a b c b 14-2$ under the control of a CaMV35S promoter to create the complemented transgenic lines. Reverse transcriptase real-time PCR (RT-PCR) and quantitative real-time PCR (qRT-PCR) using three pair of primers (located around the insertion sites, 5' regions and 3' regions, respectively) showed that the expression of $O s A B C B 14$ was $\sim 5$ folds lower in both mutant lines and $\sim 5$ folds higher in both complementation lines (osabcb14-1C and osabcb14-2C) than that in wild type (NIP) (Figure 1c,d).

AtABCB1and AtABCB19, the two closest relatives of OsABCB14 (Shen et al., 2010), were reported to transport auxin. This led us to ask whether OsABCB14 was involved in auxin 
transport. We first investigated the phenotypes of shoots and primary roots (PRs) of NIP, osabcb14 mutants and osabcb14C. The shoot and PR lengths in NIP for $7 \mathrm{~d}$ seedling were only slightly shorter than those of osabcb14-1 and osabcb14-2 under normal conditions (CK) (Figure 1e), while significantly shorter than those of osabcb14-1 and osabcb14-2 under $0.01 \mu \mathrm{M}$ 2,4-D treatment (Figure 1f). Similar results were obtained when grown in the nutritional solution containing $10 \mu \mathrm{M}$ IAA (Figure $1 \mathrm{~g}$ ). However, there was no difference between NIP and osabcb14 mutants under treatment of $0.001 \sim 1 \mu \mathrm{M}$ NAA (Figure S1). We further performed a dose-response assay to confirm the effect of 2,4-D, IAA and NAA in more details. High concentration of all the three auxins inhibited the shoot and PR growth, but more prominent in NIP than osabcb14 mutants under 2,4-D treatment at a concentration of $>10^{-8} \mathrm{~mol}^{-1}$ (Figure 1h,i) and IAA treatment at a concentration of $>10^{-7} \mathrm{~mol} \mathrm{l}^{-1}$ (Figure 1j,k), while no significant difference under NAA treatment (Figure S1). All these phenotypes in osabcb14 mutants could be reverted by transgenic lines osabcb14C (Figure 1e-k). These results confirmed that $o s a b c b 14$ mutants are insensitive to 2,4-D and IAA, but not responsive to NAA. These observations were similar to the previous studies on AUX1 (Delbarre et al., 1996; Yang et al., 2006), suggesting that OsABCB14 is required for auxin transport in either shoot or root.

\section{Expression pattern and subcellular localization of OsABCB14}

To explore the in vivo function of $O s A B C B 14$, we first analyzed the expression pattern of OsABCB14. The expression pattern of $O S A B C B 14$ was analyzed in different organs and growth stages using qRT-PCR. The results showed that $O s A B C B 14$ expression is constitutive 
in various organs. At all growth stages observed, $O S A B C B 14$ was ubiquitously expressed in all plant organs, including the root, stem, leaf, node, root-stem transition region, filling seed, panicle and flower (Figure 2a). Spatial expression analysis showed that the expression of OsABCB14 was higher in root tips than in the basal root zones (Figure 2b). GUS staining revealed that $O s A B C B 14$ is expressed in the root tip and stele of the PR and leaves. Cross section of leaf sheath showed that $O S A B C B 14$ is expressed in the vascular (Figure 2c-f). OsABCB14 is also strongly expressed in node, internode, root-stem transition region and flowers (Figure 2g-j).

To investigate the relationship between $O S A B C B 14$ and phytohormones, including auxin, the expression of $O s A B C B 14$ under various phytohormones and polar auxin transport inhibitors treatment was analyzed by qRT-PCR. As shown in Figure 2k and Figure S2a, the expression of $O s A B C B 14$ was greatly induced by 2,4-D, IAA, 6-BA, ABA, SA or NOA treatment. However, the expression of $O s A B C B 14$ was only slightly up-regulated under NAA, $\mathrm{GA}_{3}$ and NPA treatment. Furthermore, when treated by $1 \mu \mathrm{m} 2,4-\mathrm{D}$ or $10 \mu \mathrm{m}$ IAA, the expression of OsABCB14 was strongest induced after $12 \mathrm{~h}$ (Figure 21,m; Figure S2b,c).

To determine the subcellular localization of the OsABCB14 protein, OsABCB14 was cloned into $\mathrm{pH} 7 \mathrm{FWG} 2$ and that like fused with the enhanced green fluorescent protein (EGFP). Transient expression of OsABCB14 in the epidermis cells of $N$. benthamiana leaves and onion epidermis indicating that OsABCB14 was localized at the plasma membrane (Figure $3 a-c)$. 
osabcb14-1 and osabcb14-2 mutants own reduced auxin concentrations

Loss-of-function mutation of AtAUX1, which is an auxin influx transporter, resulted in reduced auxin concentration and auxin transport (Yu and Wen 2013). In addition, the loss-of-mutations of AtABCB19 identified as auxin efflux transporters also led to reduced auxin concentration and auxin transport (Noh et al., 2001). To determine whether the knockdown of OsABCB14 also alters auxin concentration and auxin transport in rice, we measured the auxin concentration and polar auxin transport in NIP and osabcbl4 mutants. The results showed that the auxin concentrations of osabcb14-1 and osabcb14-2 in both shoot and root were significantly lower than in NIP (Figure 4a,b). The staining of DR5:GUS, a biological marker to study the native auxin distribution, was observed in NIP and osabcb14 mutants (Figure 4c,d), and the results coincided with the above measurements. In addition, examination of the transcription of well-characterized auxin-responsive genes in osabcb14 mutants showed that mRNA levels of OSIAA3, OsIAA9, OsIAA23 and OSSAUR39 were down-regulated in osabcb14 mutants (Figure 4e,f), which is hence consistent with the reduced auxin sensitivity and auxin concentration in osabcb14 mutants.

\section{Auxin transport activity in yeast and osabcb14 mutants}

Reduced auxin sensitivities and auxin levels prompted us to quantify auxin transport capacities of OsABCB14. First, we functionally expressed OsABCB14 in the baker's yeast. Yeast expressing $O s A B C B 14$ accumulated 3-times more IAA than the vector control, indicating an auxin uptake activity for OsABCB14 (Figure 5a). Auxin uptake by OsABCB14 was specific as the diffusion control benzoic acid (BA) was not transported differently to the 
vector control. Next, we quantified auxin loading (= influx) into rice protoplasts not yet shown before. Both osabcb14 alleles revealed significantly reduced auxin loading, pointing again to an auxin import activity of OsABCB14 (Figure 5b). In order to test the facultative efflux capacity of OsABCB14 reported for Arabidopsis orthologs AtABCB4 and AtABCB21 (Terasaka et al., 2005; Yang and Murphy, 2009; Kamimoto et al., 2012; Kubes et al., 2012), we quantified also IAA efflux after loading of protoplasts. Interestingly, both osabcbl4 alleles revealed significantly enhanced IAA export indicating again a preferred import directionality for OsABCB14, that when absent reduces efflux most probably by lack of re-import of effluxed IAA (Figure 5c). In analogy to auxin treatments, no import of NAA by OsABCB14 was found known to be not dependent on auxin uptake systems as it can freely diffuse into cells (Delbarre et al., 1996; Yang et al., 2006). Finally, acropetal auxin transport of osabcb14-1 and osabcb14-2 roots was found to be decreased $\sim 59 \%$ and $63 \%$, respectively, in comparison to NIP (Figure 5d), while basipetal auxin transport was not different from NIP (Figure 5e). This suggests in summary that OsABCB14 functions as an auxin importer involved in acropetal transport but not basipetal transport of auxin.

osabcb14 mutants are insensitive to Fe deficiency and OsABCB14 was involved in iron homeostasis

TaABCB1 was reported to be involved in aluminum tolerance and calcium homeostasis in wheat (Sasaki et al., 2002). To test whether OsABCB14 is also functionally related to ion homeostasis, we measured the metal ion concentrations in the seeds, shoots and roots of NIP 
and osabcb14 mutants. As shown in Figure 6a, the Fe concentrations in the seeds, shoots and roots of the two osabcbl4 mutant lines were all significantly higher $(56 \%$ higher in osabcb14-1 and 43\% higher in osabcb14-2 for seeds; $51 \%$ higher in osabcb14-1 and 38\% higher in osabcb14-2 for shoots; 35\% higher in osabcb14-1 and 40\% higher in osabcb14-2 for roots) than that in NIP. The concentrations of the other metals, such as $\mathrm{Mn}, \mathrm{Cu}, \mathrm{Zn}$, and Mg were also enhanced in the osabcb14 mutants than in NIP (Figure S3). These results suggested that OsABCB14 might be involved in ion homeostasis.

To understand the relationship between OsABCB14 and $\mathrm{Fe}$ homeostasis, we further investigated the phenotypes of osabcb14 mutants under iron-deficient nutrient solution $(-\mathrm{Fe})$. osabcb14 mutants displayed significantly longer shoots and roots than NIP under $-\mathrm{Fe}$ conditions, but only slightly longer roots than NIP and normal shoot length under CK (Figure 6b-d). The chlorophyll concentration of osabcb14 mutants was significantly higher $(161 \%$ in osabcb14-1 and 183\% in osabcb14-2 ) than that of NIP under -Fe (Figure 6e). In addition, the differences of Fe concentration between NIP and osabcbl4 mutants were more obvious under $-\mathrm{Fe}$ treatment $(65 \%$ and $40 \%$ higher in shoots and roots of osabcb14-1, 40\% and $43 \%$ higher in shoots and roots of osabcb14-2) (Figure 6f-g) compared to CK (Figure 6a). These results suggest that $o s a b c b 14$ mutants are insensitive to $-\mathrm{Fe}$.

The enhanced Fe concentration in both shoots and roots of osabcb14 mutants under CK and -Fe may have been due to enhanced Fe concentration in the seeds or the impaired Fe uptake 
or homeostasis or both of them. To evaluate the effect of $O s A B C B 14$ disruption on the rice Fe uptake and signaling system, we analyzed the expression level of Fe-deficiency responsive genes IRT1 (Fe-regulated transporter 1), IRT2, IRO2 (Fe-responsive operator 2), YSL15 (yellow-stripe like 15), NAAT1 (nicotianamine aminotransferase 1), NRAMP2 (natural resistance-associated macrophage protein 2), NAS1 (nicotianamine synthase) and NAS2. The results showed that the expression of all these genes was up-regulated in osabcb14 mutants (Figure 7a, S4a). These genes were reported to be up-regulated under-Fe (Jia et al., 2011; Qi et al., 2012). Consisted with that, qRT-PCR analysis here showed that all these genes were induced in NIP and osabcb14 mutants under $-\mathrm{Fe}$, but the induced folds in osabcb14 mutants were significantly lower than that in NIP (Figure 7b; Figure S4b). This result closely coincided with the observations above - osabcb14 mutants are insensitive to Fe deficiency.

\section{DISCUSSION}

In the rice genome, $22 A B C B$ genes have been identified (Shen et al., 2010), but not one of them has been functionally characterized. Here, we report the biological function of OsABCB14 in auxin uptake and iron homeostasis in rice.

Evidence presented in this and other articles demonstrates that many $\mathrm{ABCB}$ family members play critical roles in auxin-dependent development processes. For example, research on AtABCB1 and AtABCB19, whose overexpression and disruption led to de-regulation of root elongation, gravitropism and phototropism (Noh et al., 2003; Lewis et al., 2007; Wu et al., 2007), suggests that they are involved in auxin-dependent programs. Several studies also 
supported the role for AtABCB4 in auxin-mediated developmental process, mainly in root elongation (Santelia et al., 2005; Terasaka et al., 2005). OsABCB14 as the closest rice ortholog of AtABCB1 and AtABCB19, conferred altered sensitivity to high concentrations of 2,4-D and IAA (Figure 1e-k), suggesting its involvement in auxin-mediated program.

Reduced responses of osabcb14-1 and osabcb14-2 to 2,4-D and IAA are similar to the previous studies on AtAUX1 (Yang et al., 2006). 2,4-D as a synthetic auxin that is transported into plant cells predominantly by a carrier (Delbarre et al., 1996; Yamamoto and Yamamoto, 1998), can serve very well to assess the activity of auxin uptake carriers (Hoyerova et al., 2008). A defect in the auxin uptake carrier is expected to lead to a decreased sensitivity to high concentrations of 2,4-D and both osabcb14 mutants exhibited that phenotype, suggesting that $O S A B C B 14$ may play an important role in the influx phase of polar auxin transport. These data are supported by the finding that osabcb14 shows an unaltered sensitivity to the synthetic auxin, NAA (Figure S1), known to be independent of an uptake system, such as AUX1 (Delbarre et al., 1996; Yamamoto and Yamamoto, 1998) or ABCB4-like ABCBs (Kamimoto et al., 2012).

Results of IAA transport analysis from yeast and leaf protoplasts prepared from knock-down rice mutants alleles verify that OsABCB14 functions as an auxin transporter and are in complete accordance with an uptake or import directionality (Figure 5). This finding is slightly surprising because OsABCB14 clusters closer to Arabidopsis ABCB19 (and ABCB1) than to $\mathrm{ABCB} 4 / \mathrm{ABCB} 21$ that was characterized as exporter and facultative im/exporters, 
respectively. However, our data (especially efflux experiments from rice protoplasts; Figure 5c) indicate that OsABCB14 functions under tested conditions primarily as importer. Therefore these data also suggest that transport directionalities cannot simply be deduced by phylogenetic analyses or that transport directionalities are achieved differently in monocots and dicots.

Acropetal auxin transport of both osabcb14 mutant roots was significantly lower than in NIP, suggesting that OsABCB14 functions in acropetal (root-ward) polar auxin transport. As a result knock-down roots of $O S A B C B 14$ displayed reduced auxin concentrations and DR5:GUS activity (Figure 4a-d), most probably caused by reduced auxin delivery from the shoot. Interestingly, also shoot IAA levels were strongly reduced in both mutant alleles suggesting altered shoot transport. However, we failed to quantify shoot transport in osabcb14 alleles reliably because measurements of shoot transport are still a technical challenge in rice. However, we speculate that like in roots probably also acropetal (= shoot-ward) auxin transport might be reduced in osabcb14 shoots. Reduced acropetal PAT capacities and auxin levels in the mutant, however, are also in agreement with described roles for AUX1 in acropetal auxin transport in the Arabidopsis root (Yu and Wen 2013). Therefore, this result also adds to the growing evidences supporting a major role of $\mathrm{ABCBs}$ in polar auxin transport.

In summary, our study provides convincing evidence for OsABCB14 as a plasma membrane auxin influx transporter (Figure 1, 3, 4, 5). However, we surprisingly found that OsABCB14 
is also involved in ion homeostasis. $\mathrm{Fe}, \mathrm{Mn}, \mathrm{Cu}, \mathrm{Zn}, \mathrm{Mg}$ and $\mathrm{Ca}$ are important nutrient element for plants growth and development. Fe concentration of osabcb14 mutants in shoots, roots and seeds were significantly enhanced (Figure 6a) and the expression level of Fe-deficiency responsive genes was significantly up-regulated in osabcb14 mutants (Figure 7a; Figure S4a). Thus, increased Fe concentrations in shoot and root of osabcb14 mutants (fourteen-day old) due to not only the higher Fe concentrations in seeds but also the up-regulated expression level of Fe-deficiency responsive genes. In addition, osabcb14 mutants displayed insensitive phenotype and less reduced gene expression level to $-\mathrm{Fe}$ (Figure 6b-e, 7b; Figure S4b) and increased $\mathrm{Mn}, \mathrm{Cu}, \mathrm{Zn}$ and $\mathrm{Mg}$ concentration (Figure S3). Together, these results suggest that disruption of $O s A B C B 14$ could affect negatively iron and homeostasis of other metal ions. Seeds of osabcb14 mutants have higher Fe concentration (56\% higher in osabcb14-1 and 43\% higher in osabcb14-2) (Figure 6a), indicating that osabcb14 mutants maybe can be used for creating iron-enriched crops. In the dicot Arabidosis, auxin importer AUX1 was also shown to be involved in Fe deficiency and the enhanced elongation of lateral roots in response to local Fe was demonstrated to depend on AUX1 action (Chen et al., 2010, Giehl et al., 2012). Thus, OsABCB14 may affect ion transport though the regulation of down-stream ion transporters. A systematic transcriptome analysis of osabcb14 mutants grown under $\mathrm{CK}$ and $-\mathrm{Fe}$ will elucidate (out) related genes in Fe and auxin signaling systems. 


\section{EXPERIMENTAL PROCEDURES:}

\section{Plant materials and growth conditions}

Rice plants (Oryza sativa L.) were grown in normal culture solution containing $1.425 \mathrm{mM}$ $\mathrm{NH}_{4} \mathrm{NO}_{3}, 0.323 \mathrm{mM} \mathrm{NaH}_{2} \mathrm{PO}_{4}, 0.513 \mathrm{mM} \mathrm{K}_{2} \mathrm{SO}_{4}, 0.998 \mathrm{mM} \mathrm{CaCl}_{2}, 1.643 \mathrm{mM} \mathrm{MgSO}, 0.25$ $\mathrm{mM} \mathrm{NaSiO}{ }_{3}, 0.009 \mathrm{mM} \mathrm{MnCl}_{2}, 0.019 \mu \mathrm{M} \mathrm{H}_{3} \mathrm{BO}_{3}, 0.152 \mu \mathrm{M} \mathrm{ZnSO}_{4}, 0.155 \mu \mathrm{M} \mathrm{CuSO}_{4}, 0.075$ $\mu \mathrm{M}\left(\mathrm{NH}_{4}\right)_{6} \mathrm{Mo}_{7} \mathrm{O}_{24}$ and $0.125 \mathrm{mM}$ EDTA-Fe (II) (Yoshida et al., 1976) without (CK) or with treatments, $\mathrm{pH}=5.2 \sim 5.5$. Rice plants were grown in growth chambers with $60-70 \%$ humidity and a light/dark cycle of $12 / 12 \mathrm{~h}$ at $30 / 24^{\circ} \mathrm{C}$. For transient expression, Nicotiana benthamiana plants were grown in vermiculite containing Murashige and Skoog salt nutritional liquid in a growth chamber with $60-70 \%$ humidity and a light/dark cycle of $12 / 12 \mathrm{~h}$ at $25 / 18^{\circ} \mathrm{C}$.

\section{Identification of the osabcb mutants}

Two TOS17 insertion lines for OsABCB14, osabcb14-1 (NF6030) and osabcb14-2 (NG3129), were obtained from the Rice Genome Resource Center in Japan. The homozygous lines was screened by PCR, using primer TOS17-tail6 to confirm the integration of TOS17 in two mutant lines and gene-specific primers $\mathrm{ABCB} 14-1 \mathrm{U} / \mathrm{L}$ and $\mathrm{ABCB} 14-2 \mathrm{U} / \mathrm{L}$ to identify wild-type (NIP)-bound OsABCB14. The PCR insertion products were ligated with pMD19-T Simple Vector (TaKaRa, Japan) and transformed into Escherichia coli DH5 $\alpha$, and the flanking sequences of the TOS17 insertion site were sequenced by Invitrogen (Shanghai, China). To confirm that the transcription level of the $O s A B C B 14$ gene in the NIP and two TOS17 homozygous lines, RT-PCR was performed using three pairs of primers, RTABCB14U1/L1, RTABCB14U2/L2 and RTABCB14U3/L3. qRT-PCR was performed 
using another three pairs of primers, qRTABCB14U1/L1, qRTABCB14U2/L2 and qRTABCB14U3/L3. Primer sequences for the PCR and RT-PCR are listed in Table S1.

\section{Vector construction}

The OsABCB14 (Os04g38570) coding region was amplified from the full-length cDNA (AK103526) using primers ABCB14-OU/L (Table S1) for cloning into pH7FGW2 to create ABCB14: EGFP fusion construct according to the manufacturer's instructions (Invitrogen). The final construct 35S:ABCB14-EGFP was transiently expressed in the leaves of $N$. benthamiana plants and onion as previously described (Qi et al., 2012). After 2d, the fluorescence was visualized under the confocal microscopy (Zeiss Leica TCS SP5; Mannheim, Germany) as described (Guo et al., 2003). These constructs were also introduced into Agrobacterium tumefaciens strain EHA105 using electroporation and transferred into osabcb14-1 and osabcb14-2 using callus infection method (Hiei et al., 1994) for complementation constructs. To analysis the transcription level of the $O s A B C B 14$ gene in the complementation lines, the primers RTABCB14U1/L1 and qRTABCB14U1/L1 listed in Table S1 were used.

To construct ProOsABCB14:GUS, a $2.1 \mathrm{~kb}$ promoter region of the OsABCB14 gene was amplified by PCR using primers ABCB14-proU/L (listed in TableS1) for cloning into SalI-KpnI site of pBI101.3-GUS-plus. The final construct ProOsABCB14:GUS was introduced into Agrobacterium tumefaciens strain EHA105 using electroporation and transferred into NIP using callus infection method (Hiei et al., 1994). 


\section{Analysis of IAA concentrations and transport}

Free IAA concentrations in seedling tissues were performed as described previously (Shen $e t$ al., 2013). Briefly, $20 \mathrm{mg}$ of fresh shoots or roots of seven-day seedlings grown on normal nutrient solution were washed by sterile deionized water several times. The samples were then ground into fine powder in liquid nitrogen and dissolved in $50 \mathrm{mM} \mathrm{KH} 2 \mathrm{PO} 4-\mathrm{NaOH}$ with $0.02 \%\left(\mathrm{w} / \mathrm{v}\right.$ ) ascorbic acid, then $250 \mathrm{pg}$ of ${ }^{13} \mathrm{C}$-IAA were added to each sample solution. Free IAA concentrations were measured by gas chromatography-selected reaction monitoring mass spectrometry. The DR5:GUS construct was transformed into NIP and osabcb14 mutants to detect auxin distribution in T2 generation. For staining of DR5:GUS seedlings, $100 \mathrm{mM}$ sodium phosphate buffer (PH7.0) containing $\quad 0.1 \%$ v/v Triton X-100 and 2mM X-Gluc was used. Tissues were vacuum infiltrated for 15 min with staining solution and incubated for 30 min at $37^{\circ} \mathrm{C}$ before being observed using a Zeiss Leica TCS SP5 (Mannheim, Germany). Analyses of polar ${ }^{3} \mathrm{H}-\mathrm{IAA}$ transport were performed according to Qi et al., (2008).

Yeast auxin loading experiments were performed as in Kamimoto et al (2012). In brief, JK93da transformants were grown to $\mathrm{OD} 600=1$, washed and incubated at $30^{\circ} \mathrm{C}$ with 1 $\mathrm{ml} / \mathrm{ml}$ 5-[ $\left.{ }^{3} \mathrm{H}\right] \mathrm{IAA}$ (specific activity $7.4 \times 10^{11} \mathrm{~Bq} / \mathrm{mmol}$, American Radiolabeled Chemicals) and $\left[{ }^{3} \mathrm{H}\right] \mathrm{BA}\left(9.3 \times 10^{11} \mathrm{~Bq} / \mathrm{mmol}\right.$, American Radiolabeled 60 Chemicals) in SD media $(\mathrm{pH}$ 5.5). Aliquots of $1 \mathrm{ml}$ were filtered twice with cold water after 0 and 10 minutes, respectively, and the retained radioactivity was quantified by scintillation counting. Rice protoplasts were prepared as described in Zhang et al (2011) with the exception that leaf digests were performed over-night. Transport assays were performed as described in Henrichs et al (2012). 
In brief, intact protoplasts were loaded by incubation with $1 \mathrm{ul} / \mathrm{ml}\left[{ }^{3} \mathrm{H}\right] \mathrm{IAA}$ (specific activity $7.4 \times 10^{11} \mathrm{~Bq} / \mathrm{mmol}$, American Radiolabeled Chemicals $)$ and $4-\left[{ }^{3} \mathrm{H}\right] \mathrm{NAA}\left(9.3 \times 10^{11}\right.$ $\mathrm{Bq} / \mathrm{mmol}$, American Radiolabeled Chemicals) in the presence of $100 \mathrm{nM}$ IAA on ice. Import was started by incubation at $25^{\circ} \mathrm{C}$ and halted after 10 minutes by silicon oil centrifugation. For export assays, loading was performed for $10 \mathrm{~min}$ on ice, allowing equal loading and external radioactivity was removed by Percoll gradient centrifugation. Imported/exported radioactivity was determined by scintillation counting of the protoplast interfaces/supernatants, respectively, and is presented as the relative export/import of the initial export/uptake (export/import prior to temperature incubation).

\section{Metal ion concentrations assay}

Rice seedling grown in hydroponics to two-weeks old and peeled rice seeds were analyzed to determine their metal ion concentrations with inductively coupled plasma mass spectrometry (Agilent 7500ce; Agilent Technologies, Palo Alto, CA, USA) as previously described (Jia et al., 2011). Five biological replicates were performed for each sample.

\section{Measurement of chlorophyll concentrations}

The leaves of NIP and osabcb14 mutants grown in CK and -Fe for two weeks were collected and weighed for their fresh weight. Chlorophyll was extracted with ethanol for $24 \mathrm{~h}$ and the concentration was assayed based on the absorbance of the extract at 645, 652 and $663 \mathrm{~nm}$ (Wintermans and De Mots, 1965). 


\section{RT-PCR and qRT-PCR}

The methods of total RNA extraction, reverse transcription, and RT-PCR were as found in a previous report (Qi et al., 2012). Primer sequences for auxin-responsive genes and Fe-deficiency responsive genes are listed in Table S2 and Table S3, respectively.

\section{ACKNOWLEDGEMENTS:}

This work was supported by the National Science and Technology Support Plan (2012BAC09B01), the National Natural Science Foundation of China $(31271692,31171462$ and 31371591), the Natural Science Foundation for Distinguished Young Scholars of ZheJiang province, China (LR13C130002), the State Key Laboratory of Molecular Developmental Biology, China and funds of the Novartis Foundation and the Swiss National Funds (to M.G.). We gratefully acknowledge Professor Akio Miyao in the RGRC (Rice Genome Resource Center) in Japan for providing the full-length cDNA clones of $O s A B C B 14$, osabcb14-1 and osabcb14-2 mutants and technical assistance of Laurence Charrier.

\section{SUPPORTING INFORMATION}

Figure S1. Phenotype of osabcb14 mutants and complemented transgenic lines under NAA treatment.

Figure S2. Expression of $O s A B C B 14$ in roots responded to various treatments.

Figure S3. $\mathrm{Mn}, \mathrm{Cu}, \mathrm{Zn}, \mathrm{Mg}$ and $\mathrm{Ca}$ concentrations $(\mu \mathrm{g} / \mathrm{g})$ in leaf and root of osabcbl4 
mutants.

Figure S4. Relative expression of genes related to Fe uptake and transport in roots of NIP and osabcb14 mutants.

Table S1. Primer sequences of $O S A B C B 14$.

Table S2. Primer sequences for auxin-responsive genes.

Table S3. Primer sequences for Fe-deficiency responsive genes.

\section{REFERENCES}

Abel, S., and Theologis, A. (2010) The odyssey of auxin. Cold Spring Harb Perspect Biol. 2, a004572.

Bailly, A., Sovero, V., Vincenzetti, V., Santelia, D., Bartnik, D., Koenig, B.W., Mancuso, S., Martinoia, E., and Geisler, M. (2008) Modulation of P-glycoproteins by auxin transport inhibitors is mediated by interaction with immunophilins. J. Biol. Chem. 28, $21817-21826$.

Bandyopadhyay, A., Blakeslee, J.J., Lee, O.R., Mravec, J., Sauer, M., Titapiwatanakun, B., Makam, S.N., Bouchard, R., Geisler, M., Martinoia, E., Friml, J., Peer, W.A. and Murphy, A.S. (2007) Interactions of PIN and ABCB auxin transport mechanisms. Biochem. Soc. Trans. 35, 137-141.

Blakeslee, J.J., Bandyopadhyay, A., Lee, O.R., Mravec, J., Titapiwatanakun, B., Sauer, 
M., Makam, S.N., Cheng, Y., Bouchard, R., Adamec, J., Geisler, M., Nagashima, A., Sakai, T., Martinoia, E., Friml, J., Peer, W.A. and Murphy, A.S. (2007) Interactions among PINFORMED and P-glycoprotein auxin transporters in Arabidopsis thaliana. Plant Cell, 19, 131-147.

Bouchard, R., Bailly, A., Blakeslee, J.J., Oehring, S.C., Vincenzetti, V., Lee, O.R., Paponov, I., Palme, K., Mancuso, S., Murphy, A.S., Schulz, B., and Geisler, M. (2006) Immunophilin-like TWISTED DWARF1 modulates auxin efflux activities of Arabidopsis P-glycoproteins. J. Biol. Chem. 281, 30603-30612.

Chen, W.W., Yang, J.L., Qin, C., Jin, C.W., Mo, J.H., Ye, T. and Zheng, S.J. (2010) Nitric oxide acts downstream of auxin to trigger root ferric-chelate reductase activity in response to iron deficiency in Arabidopsis. Plant Physiol. 154, 810-819.

Cho, M. and Cho, H.T. (2012) The function of ABCB transporters in auxin transport. Plant Signal Behav. 8, pii: e22990.

Cho, M., Lee, S.H. and Cho, H.T. (2007) P-glycoprotein4 displays auxin efflux transporter-like action in Arabidopsis root hair cells and tobacco cells. Plant Cell, 19, 3930-3943.

Christie, J.M., Yang, H., Richter, G.L., Sullivan, S., Thomson, C.E. Lin, J., Titapiwatanakun, B., Ennis, M., Kaiserli, E., Lee, O.R., Adamec, J., Peer, W.A., and Murphy, A.S. (2011) phot1 inhibition of ABCB19 primes lateral auxin fluxes in the shoot apex required for phototropism. PLoS Biol. 9:e1001076.

Delbarre, A., Muller, P., Imhoff, V., and Guern, J. (1996) Comparison of mechanisms controlling uptake and accumulation of 2,4-dichlorophenoxy acetic acid, 
naphthalene-1-acetic acid, and indole-3-acetic acid in suspension-cultured tobacco cells. Planta, 198, 532-541.

Geisler, M., Blakeslee, J.J., Bouchard, R., Lee, O.R., Vincenzetti, V., Bandyopadhyay, A., Titapiwatanakun, B., Peer, W.A., Bailly, A., Richards, E.L., Ejendal, K.F., Smith, A.P., Baroux, C., Grossniklaus, U., Müller, A., Hrycyna, C.A., Dudler, R., Murphy, A.S. and Martinoia, E. (2005) Cellular efflux of auxin catalyzed by the Arabidopsis MDR/ABCB transporter AtABCB1. Plant J. 44, 179-194.

Geisler, M., Girin, M., Brandt, S., Vincenzetti, V., Plaza, S., Paris, N., Kobae, Y., Maeshima, M., Billion, K., Kolukisaoglu, U.H., Schulz, B., and Martinoia, E. (2004) Arabidopsis immunophilin-like TWD1 functionally interacts with vacuolar ABC transporters. Mol. Biol. Cell, 15, 3393-3405.

Geisler, M., Kolukisaoglu, H.U., Bouchard, R., Billion, K., Berger, J., Saal, B., Frangne, N., Koncz-Kalman, Z., Koncz, C., Dudler, R., Blakeslee, J.J., Murphy, A.S., Martinoia, E. and Schulz, B. (2003) TWISTED DWARF1, a unique plasma membrane-anchored immunophilin-like protein, interacts with Arabidopsis multidrug resistance-like transporters AtABCB1 and AtABCB19. Mol. Biol. Cell, 14, 4238-4249.

Giehl, R.F., Lima, J.E. and von Wirén, N. (2012) Localized iron supply triggers lateral root elongation in Arabidopsis by altering the AUX1-mediated auxin distribution. Plant Cell, 24, $33-49$.

Guo, F., Young, J. and Crawford, N. (2003) The nitrate transporter AtNRT1.1 (CHL1) functions in stomatal opening and contributes to drought susceptibility in Arabidopsis. Plant Cell, 15, 107-117. 
Henrichs, S., Wang, B., Fukao, Y., Zhu, J., Charrier, L., Bailly, A., Oehring, S.C., Linnert, M., Weiwad, M., Endler, A., Nanni, P., Pollmann, S., Mancuso, S., Schulz, A., and Geisler, M. (2012) Regulation of ABCB1/ABCB1-catalysed auxin transport by linker phosphorylation. EMBO J. doi: 10.1038 .

Hiei, Y., Ohta, S., Komari, T. and Kumashiro, T. (1994) Efficient transformation of rice (Oryza sativa L.) mediated by Agrobacterium and sequence analysis of the boundaries of the T-DNA. Plant J. 6, 271-282.

Hoyerová, K., Perry, L., Hand, P., Lanková, M., Kocábek, T., May, S., Kottová, J., Paces, J., Napier, R. and Zazímalová, E. (2008) Functional characterization of PaLAX1, a putative auxin permease, in heterologous plant systems. Plant Physiol. 146, 1128-1141.

Jia, L., Wu, Z., Hao, X., Carrie, C., Zheng, L., Whelan, J., Wu, Y., Wang, S., Wu, P., and Mao, C. (2011) Identification of a novel mitochondrial protein, short postembryonic roots 1 (SPR1), involved in root development and iron homeostasis in Oryza sativa. New Phytol. 189, 843-855.

Kamimoto, Y., Terasaka, K., Hamamoto, M., Takanashi, K., Fukuda, S., Shitan, N., Sugiyama, A., Suzuki, H., Shibata, D., Wang, B., Pollmann, S., Geisler, M. and Yazaki, K. (2012) Arabidopsis ABCB21 is a facultative auxin importer/exporter regulated by cytoplasmic auxin concentration. Plant Cell Physiol. 53, 2090-2100.

Krecek, P., Skupa, P., Libus, J., Naramoto, S., Tejos, R., Friml, J. and Zazímalová, E. (2009) The PIN-FORMED (PIN) protein family of auxin transporters. Genome Biol. 10, 249. 
Kubeš, M., Yang, H., Richter, G.L., Cheng, Y., Młodzińska, E., Wang, X., Blakeslee, J.J., Carraro, N., Petrášek, J., Zažímalová, E., Hoyerová, K., Peer, W.A. and Murphy, A.S. (2012) The Arabidopsis concentration-dependent influx/efflux transporter ABCB4 regulates cellular auxin levels in the root epidermis. Plant J. 69, 640-654.

Landsberg, E.C. (1981) Fe stress induced transfer cell formation: regulated by auxin? Plant Physiol. 67, 563.

Lee, M., Choi, Y., Burla, B., Kim, Y.Y., Jeon, B., Maeshima, M., Yoo, J.Y., Martinoia, E. and Lee, Y. (2008) The ABC transporter AtABCB14 is a malate importer and modulates stomatal response to $\mathrm{CO}_{2}$. Nat. Cell Biol. 10, 1217-1223.

Lewis, D.R., Miller, N.D., Splitt, B.L., Wu, G. and Spalding, E.P. (2007) Separating the roles of acropetal and basipetal auxin transport on gravitropism with mutations in two Arabidopsis multidrug resistance-like ABC transporter genes. Plant Cell, 19, 1838-1850.

Leyser, O. (2006) Dynamic integration of auxin transport and signaling. Curr. Biol. 16, R424-R433.

Mravec, J., Kubes, M., Bielach, A., Gaykova, V., Petrásek, J., Skůpa, P., Chand, S., Benková, E., Zazímalová, E. and Friml, J. (2008) Interaction of PIN and ABCB transport mechanisms in auxin distribution-dependent development. Development, 135, $3345-3354$.

Nelson, B.K., Cai, X. and Nebenführ, A. (2007) A multicolored set of in vivo organelle markers for co-localization studies in Arabidopsis and other plants.

Plant J. 51, 1126-1136.

Noh, B., Bandyopadhyay, A., Peer, W.A., Spalding, E.P. and Murphy, A.S. (2003) 
Enhanced gravi- and phototropism in plant mdr mutants mislocalizing the auxin efflux protein PIN1. Nature, 423, 999-1002.

Noh, B., Murphy, A.S. and Spalding, E.P. (2001) Multidrug resistance-like genes of Arabidopsis required for auxin transport and auxin-mediated development. Plant Cell, 13, $2441-2454$.

Peer, W.A., Blakeslee, J.J., Yang, H., and Murphy, A.S. (2011) Seven things we think we know about auxin transport. Mol. Plant, 4, 487-504.

Péret, B., Swarup, K., Ferguson, A., Seth, M., Yang, Y., Dhondt, S., James, N., Casimiro, I., Perry, P., Syed, A., Yang, H., Reemmer, J., Venison, E., Howells, C., Perez-Amador, M.A., Yun， J., Alonso， J., Beemster， G.T., Laplaze， L., Murphy， A., Bennett, M.J., Nielsen, E. and Swarup, R. (2012) AUX/LAX genes encode a family of auxin influx transporters that perform distinct functions during Arabidopsis development. Plant Cell, 24, 2874-2885.

Qi, J., Qian, Q., Bu, Q., Li, S., Chen, Q., Sun, J., Liang, W., Zhou, Y., Chu, C., Li, X., Ren, F., Palme, K., Zhao, B., Chen, J., Chen, M., and Li, C. (2008) Mutation of the rice Narrow leaf1 gene, which encodes a novel protein, affects vein patterning and polar auxin transport. Plant Physiol. 147, 1947-1959.

Qi, Y., Wang, S., Shen, C., Zhang, S., Chen, Y., Xu, Y., Liu, Y., Wu, Y. and Jiang, D. (2012) OsARF12, a transcription activator on auxin response gene, regulates root elongation and affects iron accumulation in rice (Oryza sativa). New Phytol. 193, 109-120.

Rea, P.A. (2007) Plant ATP-binding cassette transporters. Annu. Rev. Plant Biol. 58, 
$347-375$.

Romera, F.J., Lucena, C. and Alcántara, E. (2007) Plant hormones influencing iron uptake in plants. In Iron Nutrition in Plants and Rhizospheric Microorganisms (Barton, L.L., Abadía, J., Eds). The Netherlands: Springer, 251-278.

Römheld, H. and Marschner, V. (1986) Mobilization of iron in the rhizosphere of different plant species. Advances in Plant Nutrition 2, 155-204.

Sasaki, T., Ezaki, B. and Matsumoto, H. (2002) A gene encoding multidrug resistance (MDR) -like protein is induced by aluminum and inhibitors of calcium flux in wheat. Plant Cell Physiol. 43, 177-185.

Santelia, D., Vincenzetti, V., Azzarello, E., Bovet, L., Fukao, Y., Düchtig, P., Mancuso, S., Martinoia, E. and Geisler, M. (2005) MDR-like ABC transporter AtPGP4 is involved in auxin-mediated lateral root and root hair development. FEBS Lett. 579, 5399-5406.

Schikora, A. and Schmidt, W. (2001) Acclimative changes in root epidermal cell fate in response to Fe and P deficiency: a specific role for auxin? Protoplasma $\mathbf{2 1 8 , 6 7 - 7 5 . ~}$

Shen, C., Bai, Y., Wang, S., Zhang, S., Wu, Y., Chen, M., Jiang, D. and Qi, Y. (2010) Expression profile of PIN, AUX/LAX and ABCB auxin transporter gene families in Sorghum bicolor under phytohormone and abiotic stress. FEBS Lett. 277, 2954-2969.

Shen, C.J., Wang, S.K., Zhang, S.N., Xu, Y.X., Qian, Q., Qi, Y.H. and Jiang, D.A. (2013) OsARF16, a transcription factor, is required for auxin and phosphate starvation response in rice (Oryza sativa L.). Plant Cell Environ. 36, 607-620.

Shitan, N., Bazin, I., Dan, K., Obata, K., Kigawa, K., Ueda, K., Sato, F., Forestier, C. and Yazaki, K. (2003) Involvement of CjMDR1, a plant multidrug-resistance-type 
ATP-binding cassette protein, in alkaloid transport in Coptis japonica. Proc. Natl. Acad. Sci. USA, 100, 751-756.

Sidler, M., Hassa, P., Hasan, S., Ringli, C. and Dudler, R. (1998) Involvement of an ABC transporter in a developmental pathway regulating hypocotyl cell elongation in the light. Plant Cell, 10, 1623-1636.

Teale, W.D., Paponov, I. and Palme, K. (2006) Auxin in action: signalling, transport and the control of plant growth and development. Nat. Rev. Mol. Cell Biol. 7, 847-859.

Terasaka, K., Blakeslee, J.J., Titapiwatanakun, B., Peer, W.A., Bandyopadhyay, A., Makam, S.N., Lee, O.R., Richards, E.L., Murphy, A.S., Sato, F. and Yazaki, K. (2005) ABCB4, an ATP binding cassette P-glycoprotein, catalyzes auxin transport in Arabidopsis thaliana roots. Plant Cell, 17, 2922-2939.

Titapiwatanakun, B., Blakeslee, J.J., Bandyopadhyay, A., Yang, H., Mravec, J., Sauer, M., Cheng, Y., Adamec, J., Nagashima, A., Geisler, M., Sakai, T., Friml, J., Peer, W.A. and Murphy, A.S. (2009) ABCB19/ABCB19 stabilises PIN1 in membrane microdomains in Arabidopsis. Plant J. 57, 27-44.

Wang, B., Bailly, A., Zwiewka, M., Henrichs, S., Azzarello, E., Mancuso, S., Maeshima, M., Friml, J., Schulz, $\quad$ A. $\quad$ and Geisler, $\quad$ M. $\quad$ (2013) Arabidopsis TWISTED DWARF1 functionally interacts with auxin exporter ABCB1 on the root plasma membrane. Plant Cell, 25, 202-214.

Wintermans, J.F.G.M. and De Mots, A. (1965) Spectrophotometric characteristics of chlorophylls $a$ and $b$ and their pheophytins in ethanol. Biochim. Biophys. Acta 109, $448-453$. 
Woodward, A.W., Bartel, B. (2005) Auxin: regulation, action, and interaction. Ann. Bot. 95, $707-735$.

Wu, G., Lewis, D.R. and Spalding, E.P. (2007) Mutations in Arabidopsis multidrug resistance-like $\mathrm{ABC}$ transporters separate the roles of acropetal and basipetal auxin transport in lateral root development. Plant Cell, 19, 1826-1837.

Wu, T., Zhang, H.T., Wang, Y., Jia, W.S., Xu, X.F., Zhang X.Z. and Han Z.H. (2012) Induction of root $\mathrm{Fe}(111)$ reductase activity and proton extrusion by iron deficiency is mediated by auxin-based systemic signalling in Malus xiaojinensis. J. Exp. Bot. 63, $859-870$.

Yamamoto, M. and Yamamoto, K.T. (1998) Differential effects of 1-naphthaleneacetic acid, indole-3-acetic acid and 2,4-dichlorophenoxyacetic acid on the gravitropic response of roots in an auxin-resistant mutant of arabidopsis, aux1. Plant Cell Physiol. 39, 660-664.

Yang, H. and Murphy, A.S. (2009) Functional expression and characterization of Arabidopsis ABCB, AUX1 and PIN auxin transporters in Schizosaccharomyces pombe. Plant J. 59, 179-191.

Yang, Y., Hammes, U.Z., Taylor, C.G., Schachtman, D.P. and Nielsen, E. (2006) High-affinity auxin transport by the AUX1 influx carrier protein. Curr. Biol. 16, 1123-1127.

Yoshida, S., Forno, D.A., Cock, J.H. and Gomez, K.A. (1976) Routine procedures for growing rice plants in culture solution, Ed 3. International Rice Research Institute, Los Banos, Philippines.

Yu, J. and Wen, C.K. (2013) Arabidopsis aux $1^{\text {rcrl }}$ mutation 
alters AUXIN RESISTANT1 targeting and prevents expression of the auxin reporter DR5: GUS in the root apex. J. Exp. Bot. 64, 921-933.

Zhang, Y., Su, J., Duan, S., Ao, Y., Dai, J., Liu, J., Wang, P., Li, Y., Liu, B., Feng, D., Wang, J. and Wang, H. (2011) A highly efficient rice green tissue protoplast system for transient gene expression and studying light/chloroplast-related processes. Plant Methods, 7, 30 .

Figure 1. Identification of osabcb14 mutants and complemented lines.

(a) TOS17 insertion sites in osabcb14-1 and osabcb14-2. Black boxes represent the exons, and black lines represent the introns. The inverted triangle marks the insertion site.

(b) PCR analysis of the integration sites of TOS17 in osabcb14-1 and osabcb14-2. The upper bound and the lower bound indicate the $O S A B C B 14$ gene fragment and TOS17 insertion fragment, respectively.

(c) RT-PCR analysis. The upper bands show $O s A B C B 14$ gene expression (30 cycles) and the lower bands show OSACTIN gene expression (26 cycles).

(d) qRT-PCR analysis. The relative expression level of $O s A B C B 14$ gene in the mutant lines (osabcb14-1 and osabcb14-2) and the complementation lines (osabcb14-1C and osabcb14-2C). OsACTIN gene was used as an internal control. qRT-PCR experiments was analyzed using three independent biological repeats. * indicates significant difference at $\mathrm{P}<$ 0.05 and $* *$ at $\mathrm{P}<0.01$. 
(e)(f) and (g) Phenotype of osabcb14 mutants and complemented transgenic lines for seven-day seedlings under CK, 2,4-D and IAA treatments. (e) CK. (f) 2,4-D treatment. (g) IAA treatment. $\mathrm{Bar}=2 \mathrm{~cm}$.

(h)(i) Shoot length (h) and PR length (i) in osabcb14 mutants and complemented transgenic lines under various concentrations of 2,4-D treatment.

(j)(k) Shoot length (j) and PR length (k) in osabcb14 mutants and complemented transgenic lines under various concentrations of IAA treatment.

Figure 2. Expression pattern of $O S A B C B 14$.

(a) Relative expression level of $O s A B C B 14$ in each tissues of NIP at different growth stage. NIP was cultivated in normal culture solution for three weeks and transferred to field.

(b) The spatial expression of $O s A B C B 14$ in root. Roots of NIP were sampled from the root tips ( 0 to $1 \mathrm{~cm}$ ) or basal root zones ( 1 to $2 \mathrm{~cm}$ from the root tip).

(c) to (j) GUS staining in root tip (c), stele (d), leaf blade (e), cross section of leaf sheath (f), node (g), internode (h), root-stem transition region (i) and flower (j). (c)(d) and (f),

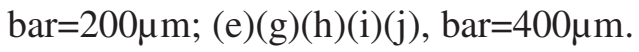


(k) $O s A B C B 14$ expression of response to various treatments. Seven-day-old seedlings grown in normal culture solution were treated for $3 \mathrm{~h}$ with $1 \mu \mathrm{M}$ 2,4-D, $10 \mu \mathrm{M}$ IAA, $1 \mu \mathrm{M}$ NAA, 1 $\mu \mathrm{M}$ 6-BA, $10 \mu \mathrm{M}$ ABA, $10 \mu \mathrm{M} \mathrm{GA}_{3}, 1 \mathrm{mM}$ SA, $1 \mu \mathrm{M}$ NPA or $10 \mu \mathrm{M}$ NOA. Total RNA from shoots were analyzed with qRT-PCR.

(1)(m) Expression of $O s A B C B 14$ in $1 \mu \mathrm{M}$ 2,4-D (1) or $10 \mu \mathrm{M}$ IAA treatments (m) at indicated time intervals. Seven-day-old seedlings grown in normal culture solution were exposed to 1 $\mu \mathrm{M}$ 2,4-D (1) or $10 \mu \mathrm{M}$ IAA treatments (m) until shoots were sampled at indicated time intervals. (a) to (m) All qRT-PCR experiments were analyzed using three independent biological repeats. OsACTIN gene was used as an internal control.

Figure 3. Subcellular localization of OsABCB14-GFP.

(a) OsABCB14-GFP fusion protein transiently expressed in tobacco. Colocalizaton of OsABCB14-GFP fusion protein with the plasma-membrane marker pm-rb CD3-1008 (Nelson et al., 2007), a fusion protein of a red fluorescent protein with a plasma membrane-localized aquaporin. Left to right: green fluorescence of OsABCB14-GFP, red fluorescence of pm-rb CD3-1008, bright-field, merged microscope images. Bar=20 $\mu \mathrm{m}$.

(b) Transient expression of the OsABCB14-GFP fusion protein in onion epidermis cells before plasmolysis. Left to right: green fluorescence of OsABCB14-GFP, bright-field, merged microscope images. Bar=50 $\mu \mathrm{m}$. 
(c) Transient expression of OsABCB14-GFP fusion protein in onion epidermis cells after plasmolysis. Left to right: green fluorescence of OsABCB14-GFP, bright-field, merged microscope images. Bar $=50 \mu \mathrm{m}$.

Figure 4. Auxin concentration in osabcbl4 mutants.

(a) Free IAA concentration in shoot of seven-day seedlings.

(b) Free IAA concentration in PR of seven-day seedlings.

(c) DR5:GUS staining in leaf sheath cross section of five-day seedlings. Bar=200 $\mu \mathrm{m}$.

(d) DR5:GUS staining in PR of five-day seedlings. Bar=200 $\mu \mathrm{m}$. Experiments in (a)(b)(c) and

(d) were analyzed using five independent biological repeats.

(e)(f) qRT-PCR analysis for auxin-responsive genes in shoots (e) and roots (f) of NIP and osabcb14 mutants of seven-day seedlings. qRT-PCR experiments were analyzed using three independent biological repeats. OSACTIN gene was used as an internal control.

\section{Figure 5. Auxin transport activity in yeast and osabcb14 mutants}

(a) Retention of IAA and of the diffusion control, benzoic acid (BA), in vector control (VC) or OsABCB14 yeast cells (strain JK93da).

(b) IAA and NAA import into NIP or osabcb14-1 and osabcb14-2 protoplasts. Values are 
mean activities \pm SEs of four individual measurements $(n=4)$.

(c) IAA and NAA export from NIP or osabcb14-1 and osabcb14-2 protoplasts. Values are mean activities \pm SEs of four individual measurements $(n=4)$.

(d) Acropetal ${ }^{3}$ H-IAA transport from the root tip $(1 \mathrm{~cm})$ of three-day seedlings.

(e) Basipetal ${ }^{3} \mathrm{H}-\mathrm{IAA}$ transport from the root tip $(1 \mathrm{~cm})$ of three-day seedlings.

Experiments were analyzed using four independent biological repeats for (a)(b)(c) and five for $(\mathrm{d})(\mathrm{e}) . *$ indicates significant difference at $\mathrm{P}<0.05$ and $* *$ at $\mathrm{P}<0.01$.

Figure 6. Fe concentration and phenotype in osabcb14 mutants.

(a) Fe concentration of seeds, shoots and roots. Left to right: seeds, shoots for fourteen-day seedlings grown in normal nutrition, roots for fourteen-day seedlings grown in normal nutrition.

(b) Phenotype of osabcb14 mutants under CK and -Fe treatments. Bar $=5 \mathrm{~cm}$.

(c)(d) Shoot length (c) and PR length (d) of fourteen-day seedlings under CK and -Fe treatments.

(e) Chlorophyll concentration of fourteen-day seedlings under CK and -Fe treatments. 
(f)(g) Fe concentration of shoot (f) and root (g) for fourteen-day seedlings under -Fe treatments (g). (a) to (g) All experiments were analyzed using five independent biological repeats. * indicates significant difference at $\mathrm{P}<0.05$ and ** at $\mathrm{P}<0.01 . \mathrm{CK}$ : normal culture solution.

Figure 7. Relative expression of genes related to Fe uptake and transport in shoots of NIP and $o s a b c b 14$ mutants.

(a) qRT-PCR analysis for genes related to Fe response in shoots of NIP and osabcb14 mutants.

(b) qRT-PCR analysis for genes related to Fe response in shoots of NIP and osabcb14 mutants under CK and -Fe. (a)(b) All qRT-PCR experiments were analyzed using three independent biological repeats. OsACTIN gene was used as an internal control. CK: normal culture solution. * indicates significant difference at $\mathrm{P}<0.05$ and $* *$ at $\mathrm{P}<0.01$. 


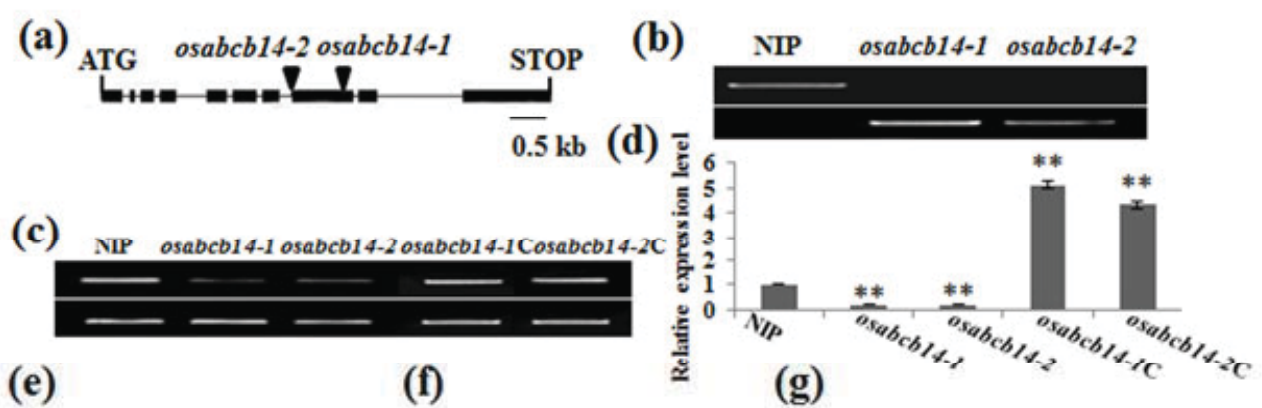

(e)

(f)

(g)
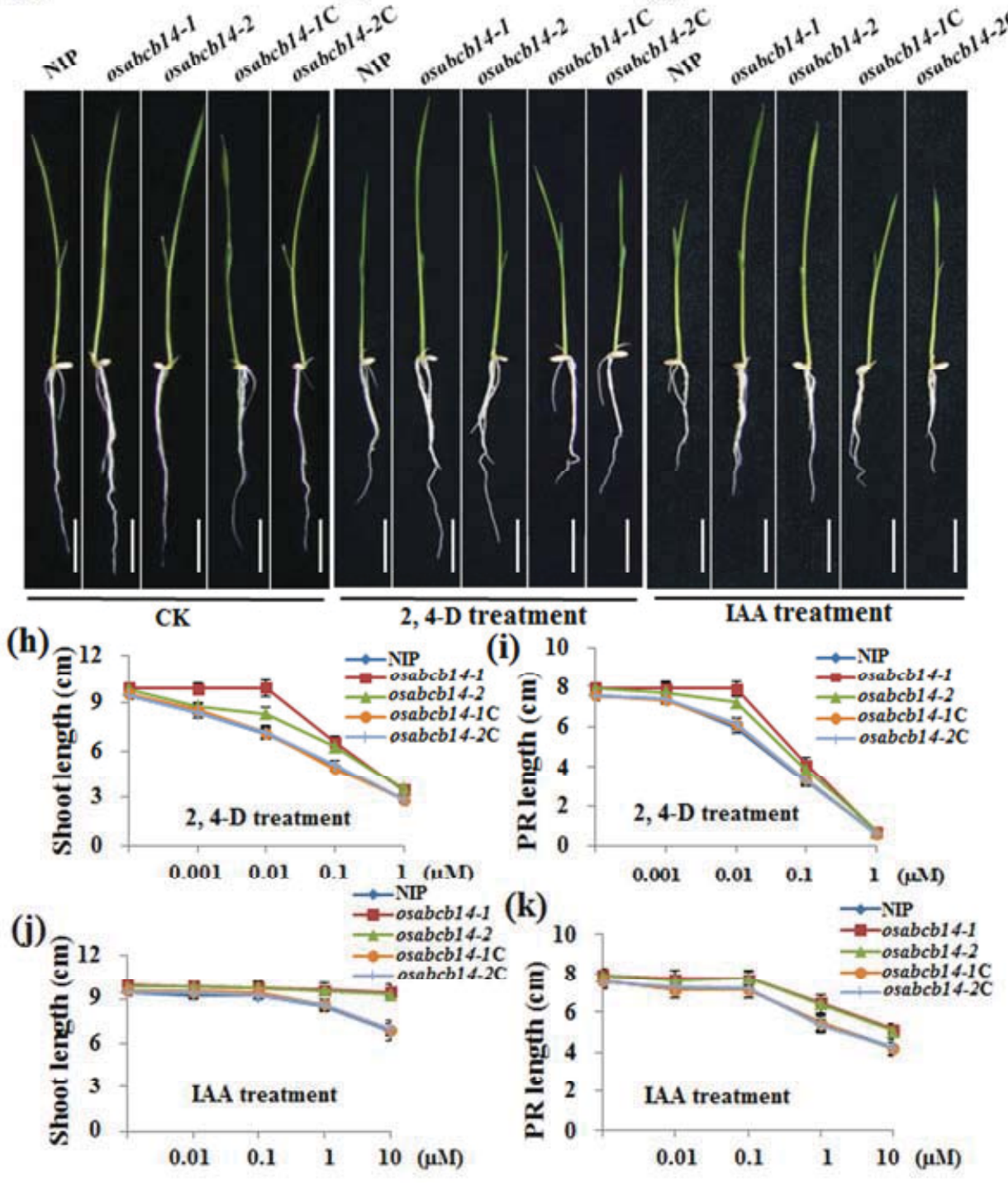


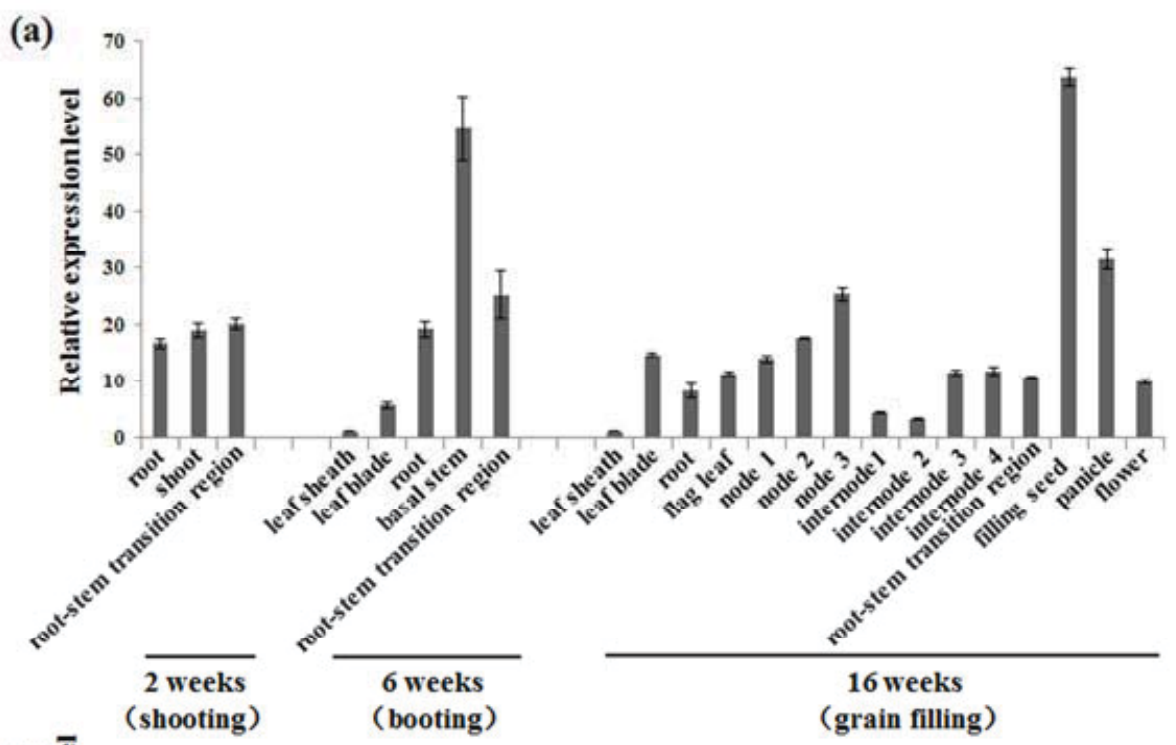

(b)
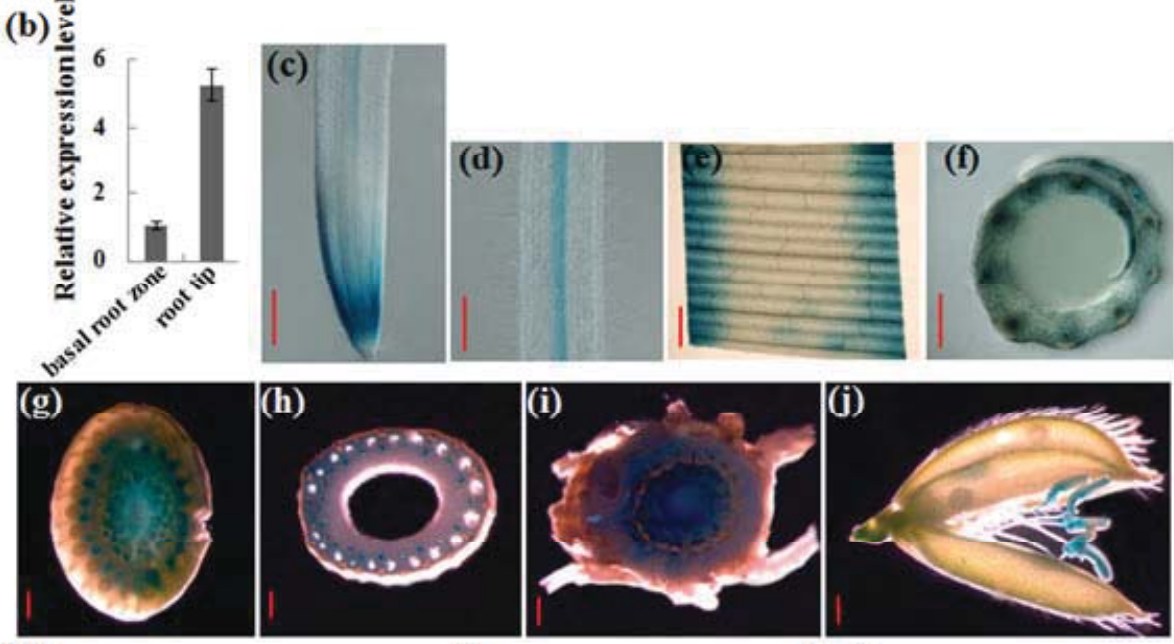

(k)

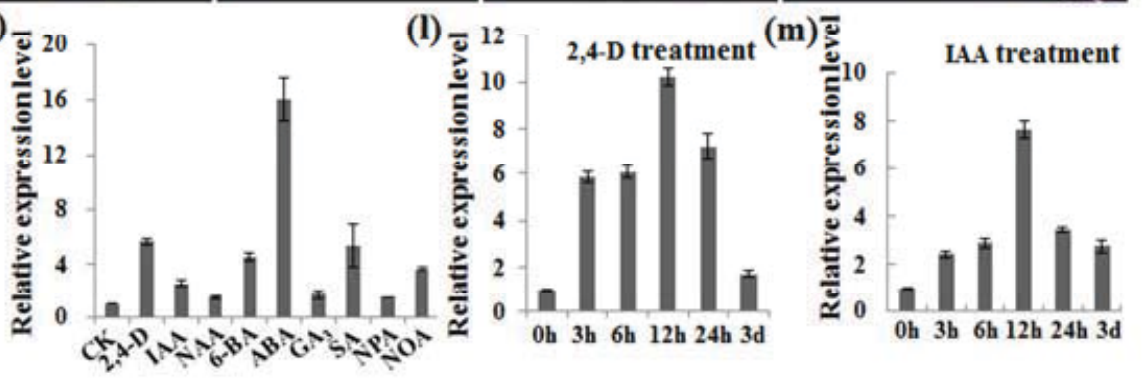


(a)

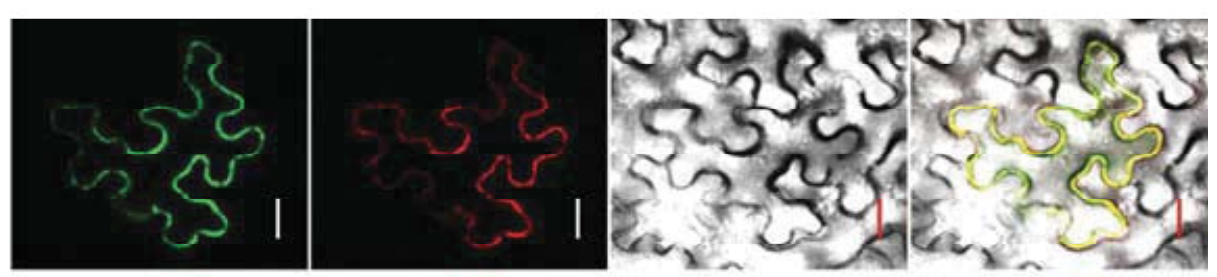

(b)

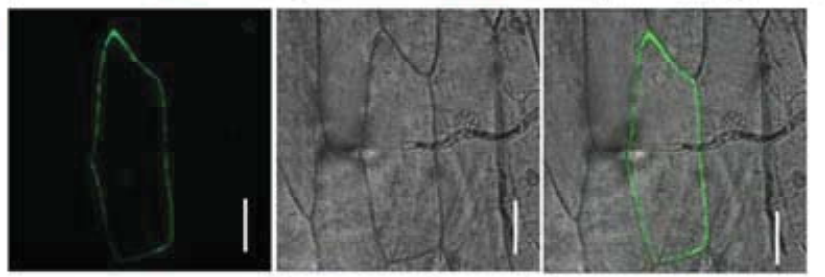

(c)

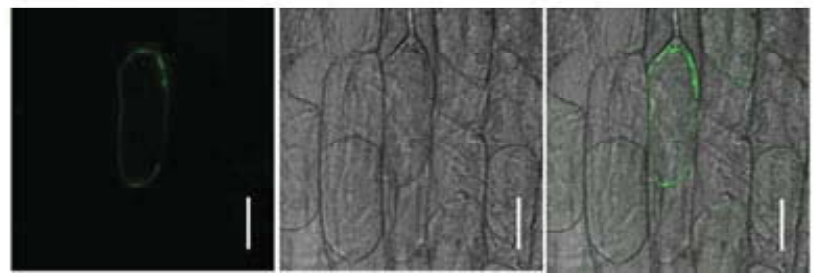

¿

'ي 


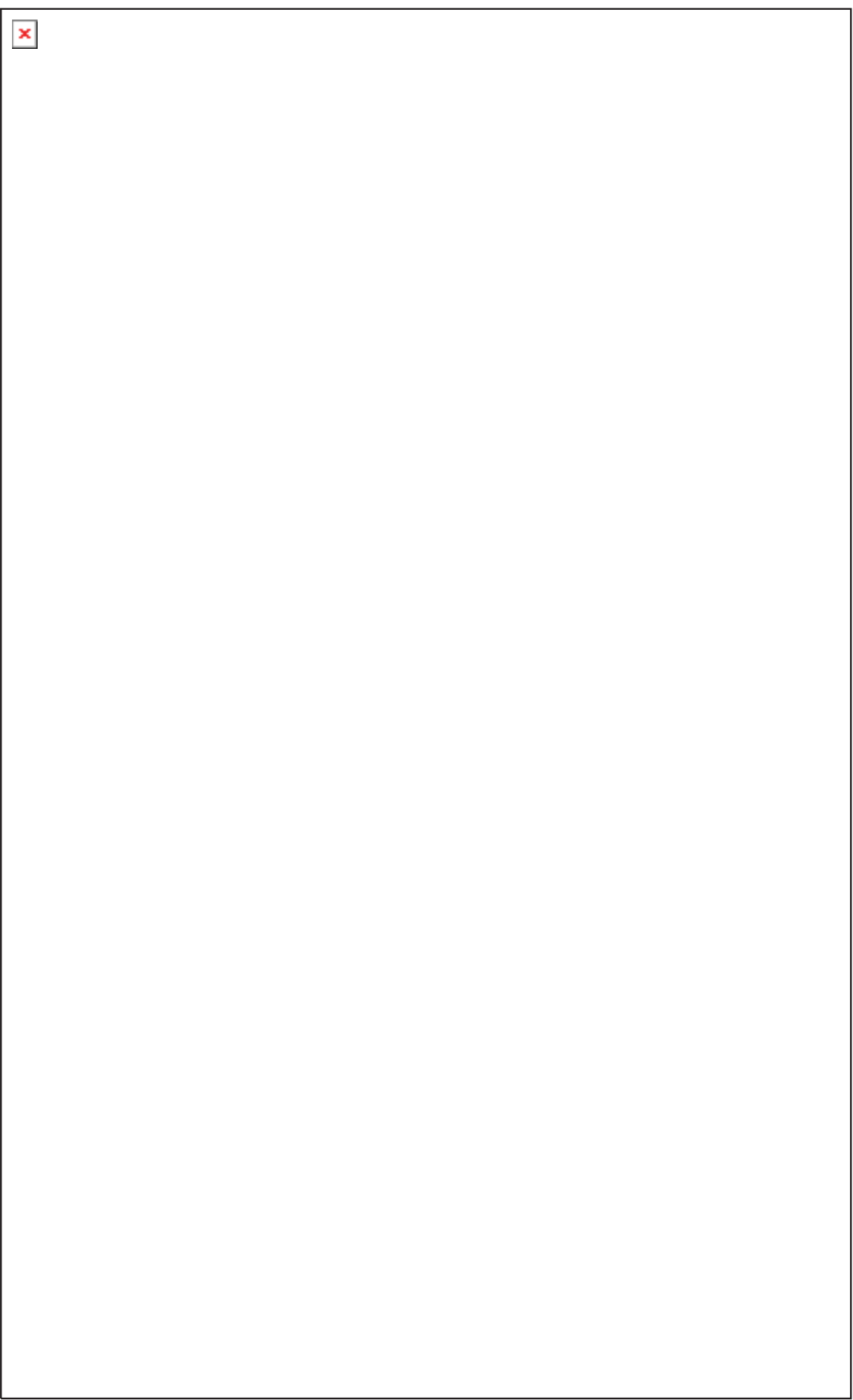


(a)

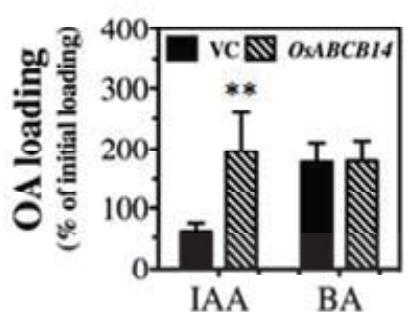

(a)

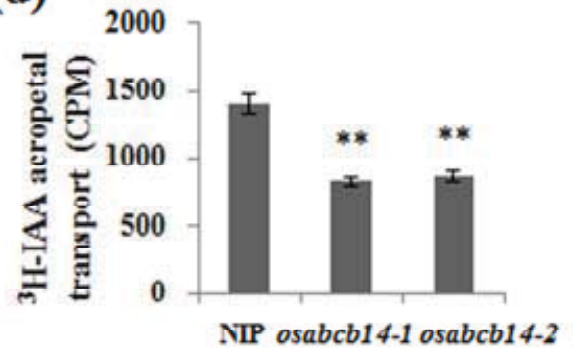

(b)

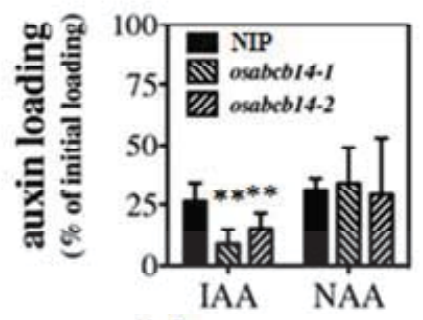

(e)

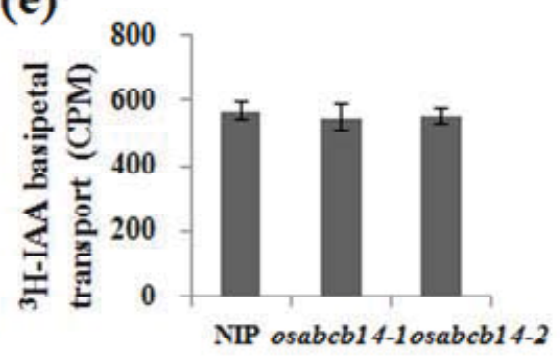

(c)

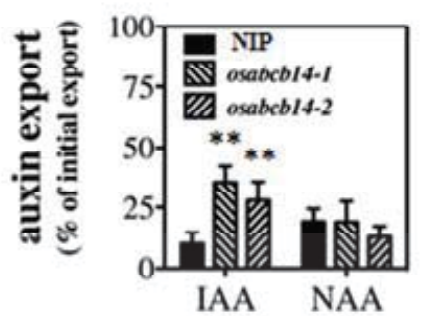

(a) 를

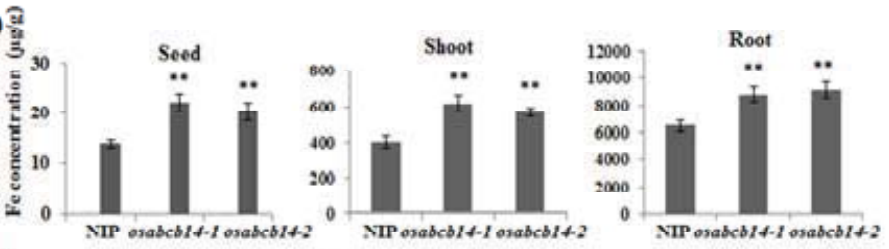

(b) CK

(c)
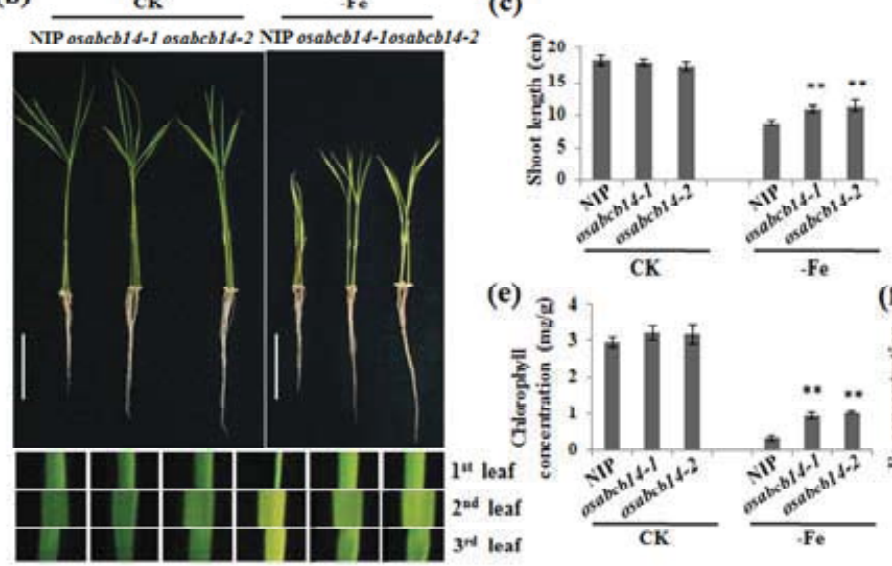

(d)
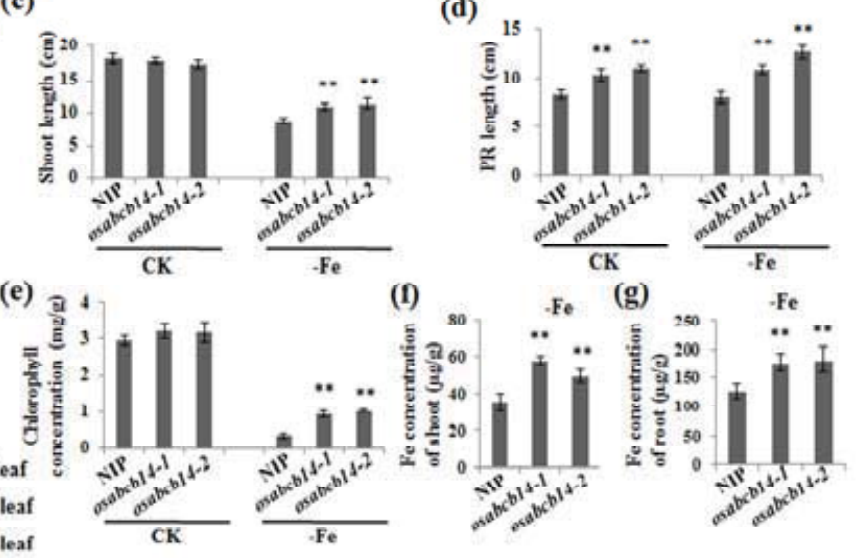


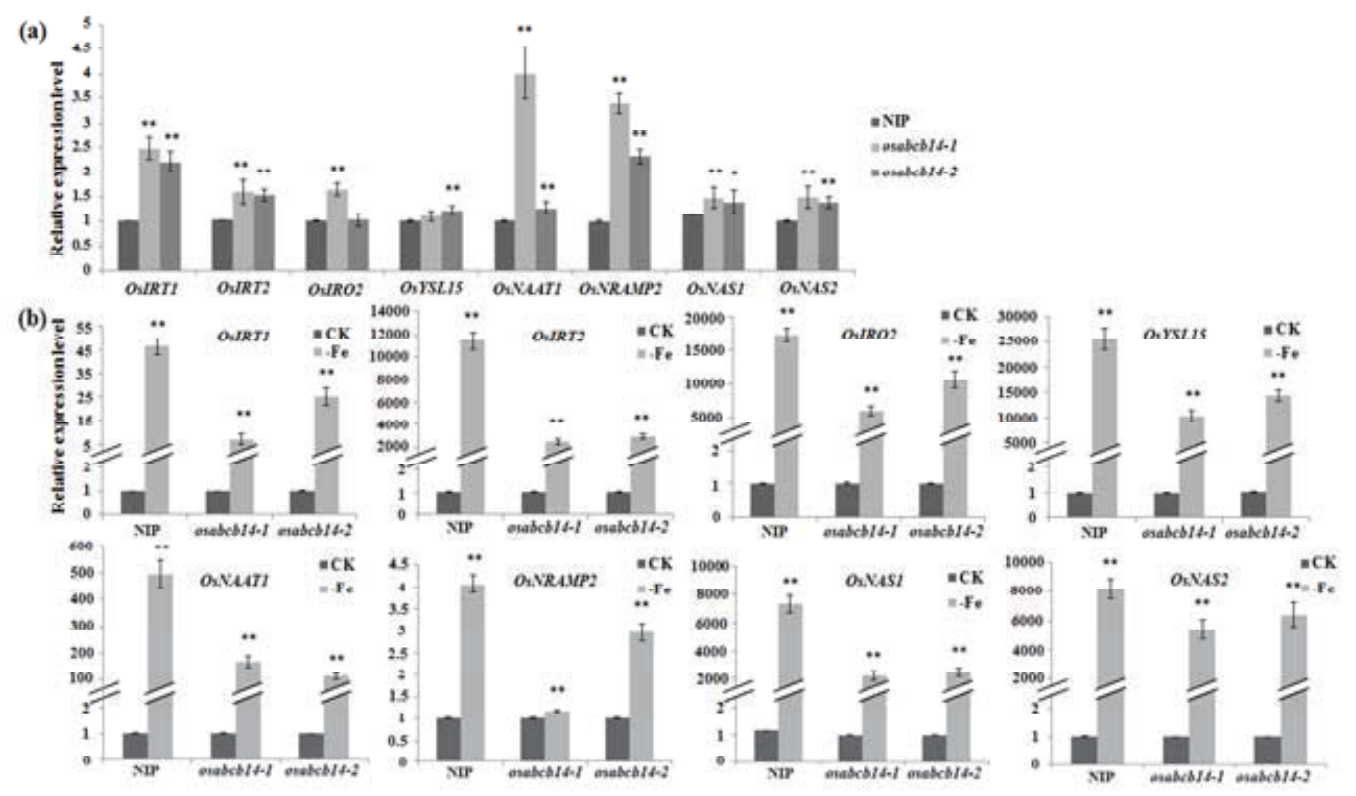

$\frac{1}{0}$
$\frac{0}{0}$
$\frac{0}{0}$
0
0
0
0 